\title{
GENUINELY MULTI-DIMENSIONAL NON-DISSIPATIVE FINITE-VOLUME SCHEMES FOR TRANSPORT
}

\author{
BRUNo DESPRÉS *, FRÉDÉRIC LAGOUTIÈRE** \\ * Commissariat à l’Énergie Atomique, DIF/DSSI \\ 91680 Bruyères-le-Châtel, France \\ e-mail: despres@cmpax.polytechnique.fr \\ ${ }^{* *}$ Université Paris-VII and Laboratoire Jacques-Louis \\ Lions, UMR 7598, 175, rue du Chevaleret, 75013 Paris, France \\ e-mail: lagoutie@math.jussieu.fr
}

\begin{abstract}
We develop a new multidimensional finite-volume algorithm for transport equations. This algorithm is both stable and non-dissipative. It is based on a reconstruction of the discrete solution inside each cell at every time step. The proposed reconstruction, which is genuinely multidimensional, allows recovering sharp profiles in both the direction of the transport velocity and the transverse direction. It constitutes an extension of the one-dimensional reconstructions analyzed in (Lagoutière, 2005; Lagoutière, 2006).
\end{abstract}

Keywords: multidimensional transport, finite-volume schemes, anti-dissipative schemes, triangular grids

\section{Introduction}

The present study ${ }^{1}$ concerns reconstruction schemes for transport equations. We are especially interested in schemes that are not dissipative, in particular for initial conditions with discontinuities. This motivates us to consider reconstructions that are not smooth, in contrast to the usual reconstructions. Indeed, we develop a scheme which consists in reconstructing the constant-in-cell datum as a discontinuous (inside each cell) function.

Typically, the goal is to develop transport schemes for mass or volume fractions in multi-fluids. These fractions can be discontinuous (at interfaces between components) or continuous (in mixing zones). A former algorithm was already developed in (Després and Lagoutière, 2007). In one dimension it was based on the limited downwind scheme (equivalent to the Ultra-bee limiter for advection with constant velocity), and the multidimensional algorithms were obtained via an alternate direction splitting strategy. The results are satisfying, in particular for interfaces (see results in the cited reference for interface instabilities in two and three dimensions). The main drawback of this method is precisely the dimensional splitting,

\footnotetext{
1 This work was partially supported by CEA/DIF Bruyères-le-Châtel.
}

which prevents the algorithm from being used on nonCartesian grids.

Here we present a way to generalize the limited downwind scheme to the case of a general triangular grid. It is based on the geometrical approach followed in (Lagoutière, 2005; Lagoutière, 2006), which provided a new interpretation of the limited downwind algorithm in terms of reconstruction schemes.

The paper is organized as follows: Sections 2 and 3 present the mathematical and numerical problems and the notation. Then we recall the reconstruction procedure leading to the limited downwind scheme in one dimension (Section 4). In Section 5, the main subject of the paper is addressed, namely, the extension of the preceding procedure to more dimensions. The presentation deals with two dimensions. Finally, Section 6 presents numerical results.

Nowadays, the most efficient algorithms for pure transport rest upon interface reconstruction, see, e.g., SLIC and Youngs' method (Noh and Woodward, 1976; Youngs, 1984) and Mosso's method (Mosso and Cleancy, 1995), which constitutes a recent promising enhancement. These methods are essentially limited to Cartesian grids. Here we try to derive a truly multidimensional reconstruction algorithm. 


\section{Model Problem}

The model considered is the linear transport equation with constant (given) velocity

$$
\left\{\begin{array}{l}
\partial_{t} u(t, x)+\operatorname{div}(\mathbf{a} u)(t, x) \\
\quad=\partial_{t} u(t, x)+\mathbf{a} \cdot \nabla_{x} u(t, x)=0 \text { for } t>0 \\
u(0, \cdot)=u^{0} \in L^{\infty}\left(\mathbb{R}^{2}\right)
\end{array}\right.
$$

where $\mathbf{a}$ is a smooth divergence-free velocity field: $\operatorname{div} \mathbf{a}(t, x)=0$ for all $(t, x) \in \mathbb{R}^{+} \times \mathbb{R}^{2}$.

The main issue for the numerical treatment of this PDE problem is the numerical diffusion. This phenomenon, easily understandable in one dimension, is much more complex in more dimensions. It is going to be decomposed into two different types of diffusion. The diffusion of the first type, which will be called the longitudinal diffusion is the one that occurs in the direction of the velocity. It is the diffusion which is present in classical one-dimensional algorithms. The diffusion of the second type, i.e., the so-called transverse diffusion, is typically multidimensional and is due to the fact that the mesh may not be aligned with the velocity. This distinction between the two phenomena could appear arbitrary, but is in accordance with the numerical tests. It is illustrated in Figs. 2 and 3 representing numerical solutions obtained with the classical upwind scheme on a square mesh with the characteristic function of a square as the initial condition (Fig. 1). The transport velocity is $\mathbf{a}=(1,0)$ (aligned with the mesh) for Fig. 2 and $\mathbf{a}=(1,1)$ (diagonal, not aligned with the mesh) for Fig. 3, and the boundary conditions on $[0,1]^{2}$ are periodic. The results are displayed for time $t=1$ (after one period).

The difference between longitudinal and transverse diffusions appears clearly in (Després and Lagoutière, 2001), which was a previous attempt to elaborate nondissipative schemes on non-Cartesian grids. Based on one-dimensional techniques, the schemes derived from this work were longitudinally anti-dissipative, but not transversely.

The new method described here is based on an interpretation of the (anti-dissipative) limited downwind scheme which was proposed in (Lagoutière, 2006) (previous works are (Després and Lagoutière, 2001; Després and Lagoutière, 2007)). This paper shows that the limited downwind scheme (in one dimension) can be understood as a reconstruction scheme, decomposed into three stages at each time step:

- a reconstruction stage, which, starting from a constant-in-cell datum, constructs a new datum, presenting in each cell one discontinuity that separates two constant values,

- a transport stage, which solves the transport operation with the new datum,

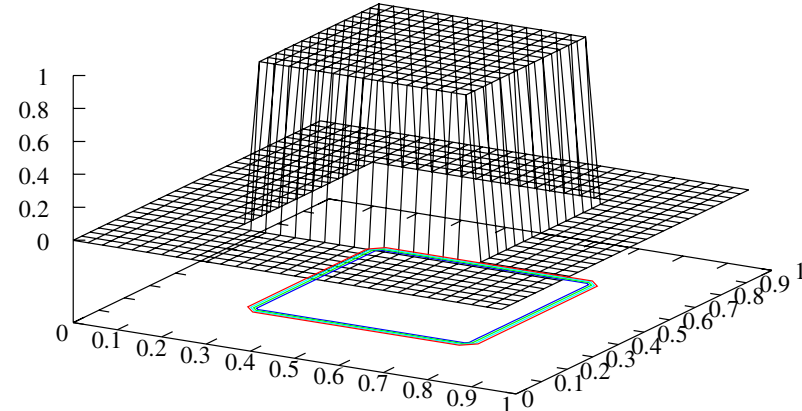

Fig. 1. Initial condition.

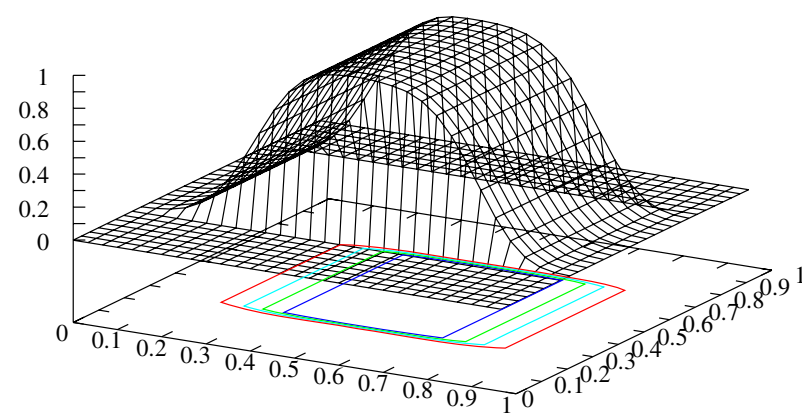

Fig. 2. Upwind scheme: $\mathbf{a}=(1,0)$, aligned with the mesh. Time $t=1$. The longitudinal diffusion applies in the direction of transport. There is no transverse diffusion.

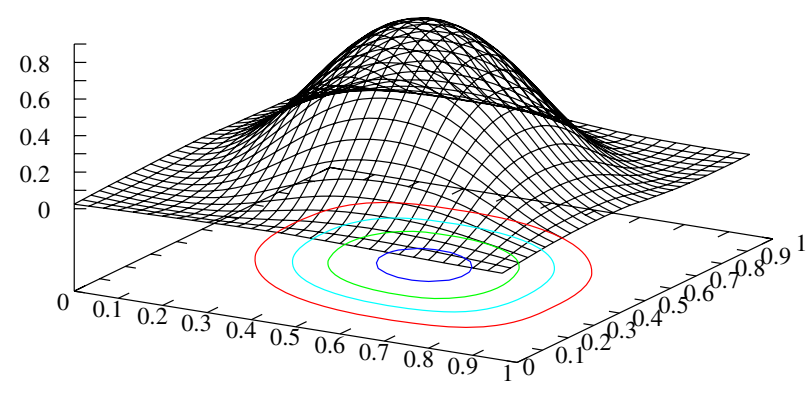

Fig. 3. Upwind scheme: $\mathbf{a}=(1,1)$, not aligned with the mesh. Time $t=1$. The diffusion which applies orthogonally to the transport direction is brought to light.

- a projection stage, which computes the mean value of the transported datum in each cell,

(see Section 4 for details of the reconstruction stage).

A natural extension of the multidimensional transport problem consists in reconstructing the datum in two steps: in the first step, a transverse reconstruction is performed, and the second step includes a longitudinal reconstruction. This is the technique which is proposed in this work. The following describes precisely these two operations.

\section{General Numerical Formalism}

This is one of finite volume methods for the problem (1). We consider a mesh of $\mathbb{R}^{2}$ composed of non-empty open 
triangles $\left(T_{j}\right)_{j \in \mathbb{Z}}$ such that $\bigcup_{j \in \mathbb{Z}} \overline{T_{j}}=\mathbb{R}^{2}$ and $T_{i} \cap T_{j}=\emptyset$ for every $i$, every $j \neq i$. For each cell $T_{j}$ (for $j \in \mathbb{Z}$ ), we denote by $K(j)$ the set of indices of neighbouring cells of $T_{j}$ (that is to say, cells having a common edge with $T_{j}$ ),

$$
K(j)=\left\{k \in \mathbb{Z} \backslash\{j\} \text { s.t. } \operatorname{meas}_{1}\left(\overline{T_{j}} \cap \overline{T_{k}}\right)>\emptyset\right\},
$$

where meas ${ }_{1}$ denotes the Lebesgue measure in one dimension. For $j \in \mathbb{Z}$ and for $k \in K(j)\left(T_{j}\right.$ and $T_{k}$ have an edge in common), we denote by $l_{j, k}$ the length of the common edge,

$$
l_{j, k}=\operatorname{meas}_{1}\left(\overline{T_{j}} \cap \overline{T_{k}}\right),
$$

and by $\mathbf{n}_{j, k}$ the unit vector normal to the common edge outward to $T_{j}$. We thus have $l_{j, k}=l_{k, j}$ and $\mathbf{n}_{j, k}=-\mathbf{n}_{k, j}$ for every $j \in \mathbb{Z}$ and every $k \in K(j)$. We then denote by $K^{+}(j)$ the set of the indices of the downwind neighbours of $T_{j}$, and by $K^{-}(j)$ the set of the indices of the upwind neighbours of $T_{j}$ :

$$
\begin{aligned}
& K^{+}(j)=\left\{k \in K(j) \text { s.t. }\left(\mathbf{a}, \mathbf{n}_{j, k}\right)>0\right\} \\
& K^{+}(j)=\left\{k \in K(j) \text { s.t. }\left(\mathbf{a}, \mathbf{n}_{j, k}\right)<0\right\} .
\end{aligned}
$$

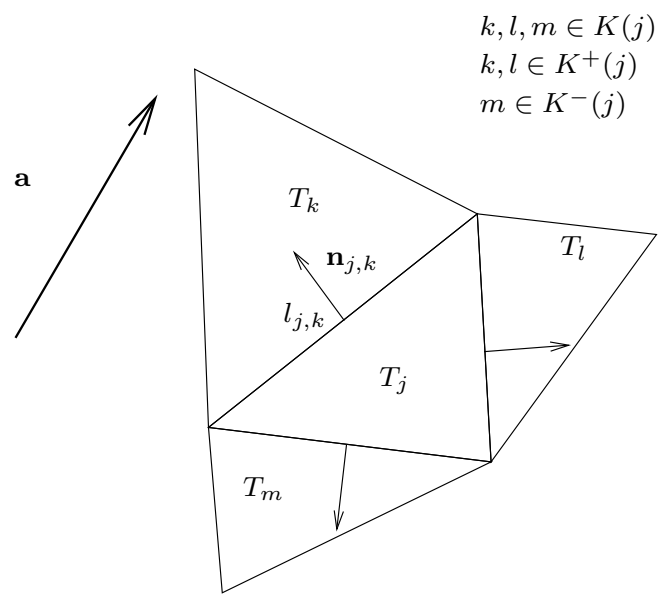

Fig. 4. Mesh and notation.

Let $s_{j}$ denote the surface of the cell $T_{j}$ :

$$
s_{j}=\operatorname{meas}_{2}\left(T_{j}\right),
$$

where meas $_{2}$ denotes the Lebesgue measure in two dimensions. The general form of the schemes considered here is obtained by choosing a time step $\Delta t>0$ and by integrating the transport equation (1) over $[n \Delta t,(n+1) \Delta t] \times T_{j}$ :

$$
\begin{aligned}
\frac{u_{j}^{n+1}-u_{j}^{n}}{\Delta t}+ & \frac{1}{s_{j}}\left(\sum_{K^{+}(j)} l_{j, k}\left(\mathbf{a}_{j, k}^{n}, \mathbf{n}_{j, k}\right) u_{j, k}^{n}\right. \\
& \left.+\sum_{K^{-}(j)} l_{j, k}\left(\mathbf{a}_{j, k}^{n}, \mathbf{n}_{j, k}\right) u_{j, k}^{n}\right)=0 .
\end{aligned}
$$

In this formula, the quantities $\mathbf{a}_{j, k}^{n}$ are approximate values of the given velocity $\mathbf{a}(t, x)$ on the edges and the $u_{j, k}^{n} \mathrm{~s}$ are approximate values of the solution on the edges between times $n \Delta t$ and $(n+1) \Delta t$. The upwind scheme is obtained taking $u_{j, k}^{n}=u_{j}^{n}$ for $k \in K^{+}(j)$ and $u_{j, k}^{n}=u_{k}^{n}$ for $k \in K^{-}(j)$. We shall propose another definition of these numerical fluxes, intended to provide more precise numerical results.

\section{Discontinuous Reconstructions in One Dimension}

In the following, for any $a, b \in \mathbb{R},\langle a, b\rangle$ denotes the interval $[a, b]$ if $a \leq b$ and the interval $[b, a]$ otherwise, i.e., we adopt the convention

$$
\langle a, b\rangle=[\min (a, b), \max (a, b)],
$$

and one has $\langle a, b\rangle=\langle b, a\rangle$.

Here we recall the principle of discontinuous reconstruction schemes in one dimension. Details and proofs can be found in (Lagoutière, 2005) with (Lagoutière, 2006). We consider a mesh (on $\mathbb{R}$ ) with a constant space step $\Delta x>0$ whose cells are the intervals $T_{j}=((j-$ $1 / 2) \Delta x,(j+1 / 2) \Delta x)$ for $j \in \mathbb{Z}$. In (Lagoutière, 2006), it is shown that for the transport equation

$$
\partial_{t} u+a \partial_{x} u=0,
$$

the limited downwind scheme of (Després and Lagoutière, 2001) is equivalent to the following three-stage scheme. Let $\left(u_{j}^{n}\right)_{j \in \mathbb{Z}}$ be a discrete datum (associated with a constant-in-cell function).

1. In each cell "reconstruct" the discrete datum in a form with more details (not constant) following the algorithm detailed above.

2. Perform the (exact) transport of this reconstructed datum at velocity $a$ for a time $\Delta t$.

3. "Project" the computed solution to obtain a new constant-in-cell datum and to be able to iterate these three operations.

It now remains to describe the reconstruction operation, the other two being trivial.

Let $\left(u_{j}^{n}\right)_{j \in \mathbb{Z}}$ be given. We define the discrete function $\bar{u}(x)=\sum_{j \in \mathbb{Z}} u_{j}^{n} \chi_{[(j-1 / 2) \Delta x,(j+1 / 2) \Delta x)}(x)$. The algorithm is defined as follows:

- if $u_{j}^{n} \notin\left(u_{j-1}^{n}, u_{j+1}^{n}\right)$, i.e., if $u_{j}^{n}$ is a local extremum, we do not reconstruct the datum in the cell $T_{j}$,

- if $u_{j}^{n} \in\left(u_{j-1}^{n}, u_{j+1}^{n}\right)$, we define

$$
\left\{\begin{array}{l}
u_{j, l}^{n}=u_{j-1}^{n}, \\
u_{j, r}^{n}=u_{j+1}^{n}, \\
d_{j}^{n}=\frac{u_{j+1}^{n}-u_{j}^{n}}{u_{j+1}^{n}-u_{j-1}^{n}},
\end{array}\right.
$$


and the reconstructed datum is defined inside $T_{j}$ by

$$
\hat{u}(x)=\left\{\begin{array}{l}
u_{j, l}^{n} \\
\text { if } x \in\left[(j-1 / 2) \Delta x,\left(j-1 / 2+d_{j}^{n}\right) \Delta x\right), \\
u_{j, r}^{n} \\
\text { if } x \in\left[\left(j-1 / 2+d_{j}^{n}\right) \Delta x,(j+1 / 2) \Delta x\right) .
\end{array}\right.
$$

Notice that when $u_{j}^{n} \in\left(u_{j-1}^{n}, u_{j+1}^{n}\right)$, the formula (3) is well defined. In particular, the denominator in the definition of $d_{j}^{n}$ is non-zero. More precisely, one always has $d_{j}^{n} \in(0,1)$, so that $\left(j-1 / 2+d_{j}^{n}\right) \Delta x \in T_{j}$. Furthermore, another essential property of this reconstruction is that it is conservative: $d_{j}^{n} u_{j, l}^{n}+\left(1-d_{j}^{n}\right) u_{j, r}^{n}=u_{j}^{n}$.

Of course, other values for $u_{j, l}^{n}$ and $u_{j, r}^{n}$ are admissible, but this choice leads to the most anti-dissipative scheme (under the constraints of $L^{\infty}$ stability and a decrease in the total variation, cf. (Lagoutière, 2006)). At last, notice that not to reconstruct the datum is equivalent to take $u_{j, l}^{n}=u_{j, r}^{n}=u_{j}^{n}$ and gives the Godunov (upwind) scheme. The discontinuous reconstruction is illustrated in Fig. 5.

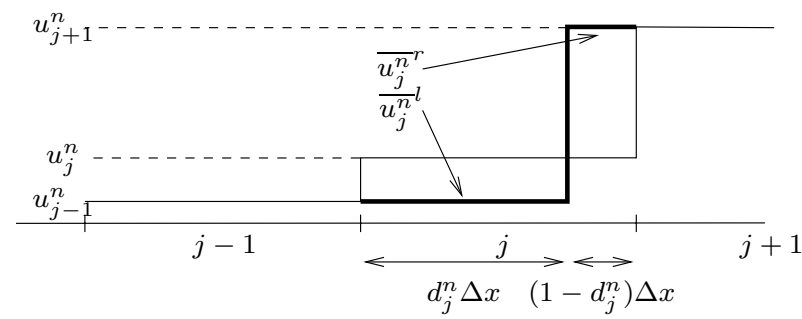

Fig. 5. Discontinuous reconstruction.

\section{Discontinuous Reconstructions in Two Dimensions}

The two-dimensional extension is not obvious. The genuine two-dimensional nature of the problem, due to the choice of a non-Cartesian grid, leads to the fact that the finite-volume method (2) cannot be seen as an exact Godunov one (which would be composed of an exact transport step, preceded by a reconstruction or not, and followed by a projection on the mesh) since only edge fluxes are taken into account, except for node fluxes. In order to separate the problems of longitudinal and transverse diffusion (see Section 1), we propose to perform the reconstruction in two steps, each being a one-dimensional reconstruction. In order to simplify the presentation, let us assume that $\mathbf{a}$ is constant in time and space. Let $\left(u_{j}^{n}\right)_{j \in \mathbb{Z}}$ be a discrete datum at time step $n$.

The first (tranverse) reconstruction boils down to splitting some cells in the direction of the velocity and modifying the value of the datum in each of these two sub-cells. The cell $T_{j}$ has at least one downwind neighbour and at most two (thanks to the divergence-free hypothesis). If it has only one downwind neighbour, we do not perform the transverse reconstruction (we do not split the cell). Let us now assume that $T_{j}$ has two downwind neighbours, $T_{k}$ and $T_{l}$. It has then one upwind neighbour, $T_{m}$. We consider the intersection point of the two edges relative to the downwind neighbours and cut $T_{j}$ along the line passing through this intersection point and parallel to $\mathbf{a}$. The two induced sub-cells are denoted by $T_{j, k}$ and $T_{j, l}: T_{j, k}$ has $T_{k}$ as its downwind neighbour, and $T_{j, l}$ has $T_{l}$ as a downwind neighbour. This cutting is illustrated in Fig. 6.

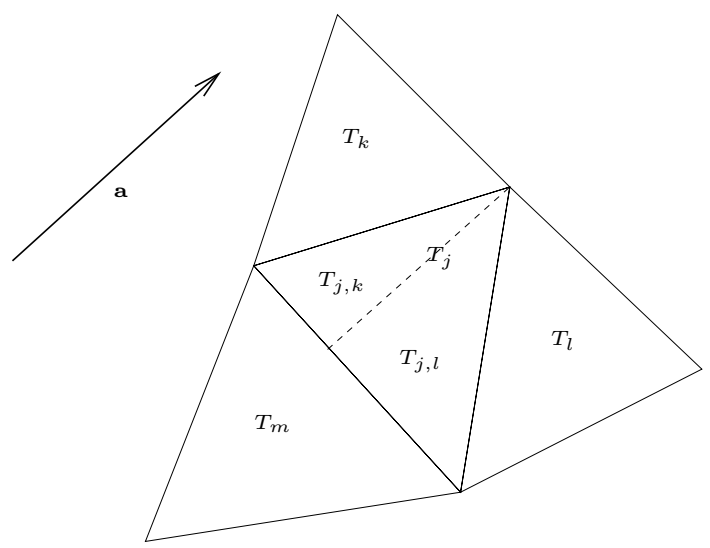

Fig. 6. Transverse reconstruction.

We denote by $s_{j, k}$ and $s_{j, l}$ the surfaces of the cells $T_{j, k}$ and $T_{j, l}$, respectively. Of course, one has $s_{j, k}+s_{j, l}=$ $s_{j}, s_{j, k}>0$ and $s_{j, l}>0$.

We now have to assign each sub-cell a value of the reconstructed solution. The adopted principle is quite similar to the one in one dimension, except that the locus of the discontinuity is determined by the geometrical aspect and not the local values of the datum: it is the line parallel to a defined just above. The aim is to define a value $u_{j, k}^{n}$ in the cell $T_{j, k}$ and a value $u_{j, l}^{n}$ in the cell $T_{j, l}$ by maximizing $\left|u_{j, l}^{n}-u_{j, k}^{n}\right|$ (to guarantee the antidissipativity) and assuring that $s_{j, k} u_{j, k}^{n}+s_{j, l} u_{j, l}^{n}=s_{j} u_{j}^{n}$ (to guarantee the conservativity). On the other hand, following the same rules as in one dimension, we impose the condition that the triplet $\left\{u_{l}^{n}, u_{j, l}^{n}, u_{j, k}^{n}\right\}$ have the same monotonicity property as the pair $\left\{u_{l}^{n}, u_{j}^{n}\right\}$ and that the triplet $\left\{u_{j, l}^{n}, u_{j, k}^{n}, u_{k}^{n}\right\}$ have the same monotonicity property as the pair $\left\{u_{j}^{n}, u_{k}^{n}\right\}$. These constraints imply in particular that the datum in $T_{j}$ would not be reconstructed if $u_{j}^{n}$ was a local extremum in the transverse direction. The algorithm is as follows:

- if $u_{j}^{n} \notin\left(u_{k}^{n}, u_{l}^{n}\right)$ (if $u_{j}^{n}$ is a local extremum in the transverse direction), we do not reconstruct $\bar{u}$ in the cell $T_{j}$ (i.e., $u_{j, l}^{n}=u_{j, k}^{n}=u_{j}^{n}$ ),

- if $u_{j}^{n} \in\left(u_{k}^{n}, u_{l}^{n}\right)$, 
- if $\left(s_{j} u_{j}^{n}-s_{j, l} u_{l}^{n}\right) / s_{j, k} \in\left[u_{j}^{n}, u_{k}^{n}\right]$, we define

$$
\begin{aligned}
& \left\{\begin{array}{l}
u_{j, l}^{n}=u_{l}^{n}, \\
u_{j, k}^{n}=\left(s_{j} u_{j}^{n}-s_{j, l} u_{l}^{n}\right) / s_{j, k} ;
\end{array}\right. \\
& \text { - if }\left(s_{j} u_{j}^{n}-s_{j, k} u_{k}^{n}\right) / s_{j, l} \in\left[u_{j}^{n}, u_{j}^{n}\right] \text {, we define } \\
& \left\{\begin{array}{l}
u_{j, l}^{n}=\left(s_{j} u_{j}^{n}-s_{j, k} u_{k}^{n}\right) / s_{j, l}, \\
u_{j, k}^{n}=u_{k}^{n} .
\end{array}\right.
\end{aligned}
$$

Lemma 1. The Courant-Friedrichs-Lewy (CFL) condition on the time step is not degraded by the transverse reconstruction.

Proof. The standard condition for the upwind scheme for the cell $T_{j}$ is

$$
\Delta t \frac{\sum_{k \in K^{+}(j)} l_{j, k}\left(\mathbf{a}, n_{j, k}\right)}{s_{j}} \leq 1,
$$

i.e., in the studied case,

$$
\Delta t \frac{l_{j, k}\left(\mathbf{a}, n_{j, k}\right)+l_{j, l}\left(\mathbf{a}, n_{j, l}\right)}{s_{j}} \leq 1 .
$$

The CFL condition for the sub-cells $T_{j, k}$ and $T_{j, l}$ is

$$
\Delta t \frac{l_{j, k}\left(\mathbf{a}, n_{j, k}\right)}{s_{j, k}} \leq 1, \quad \Delta t \frac{l_{j, l}\left(\mathbf{a}, n_{j, l}\right)}{s_{j, l}} \leq 1 .
$$

Denote by $l_{j}$ the length of the segment separating $T_{j, k}$ and $T_{j, l}$ :

$$
l_{j}=\operatorname{mes}_{1}\left(\overline{T_{j, k}} \cap \overline{T_{j, l}}\right) .
$$

One has

$$
\begin{aligned}
& s_{j, k}=\frac{l_{j} \times l_{j, k}\left(\mathbf{a}, n_{j, k}\right)}{2}, \quad s_{j, l}=\frac{l_{j} \times l_{j, l}\left(\mathbf{a}, n_{j, l}\right)}{2}, \\
& s_{j}=s_{j, k}+s_{j, l}=\frac{l_{j} \times\left(l_{j, k}\left(\mathbf{a}, n_{j, k}\right)+l_{j, l}\left(\mathbf{a}, n_{j, l}\right)\right)}{2} .
\end{aligned}
$$

The two inequalities of (5) and the inequality (4) can thus be rewritten as

$$
\frac{2 \Delta t}{l_{j}} \leq 1
$$

as they are equivalent.

Remark 1. Once the solution is reconstructed, its transport is related to one-dimensional transport since $T_{j, l}$ and $T_{j, k}$ have only one upwind and one downwind neighbour: $T_{m}, T_{l}$ and $T_{m}, T_{k}$, respectively. When $T_{j}$ has only one downwind neighbour, we do not perform the transverse reconstruction and can consider the problem as onedimensional, performing (virtually) the cutting but assigning the value $u_{j}^{n}$ to each sub-cell.
After this transverse reconstruction, the algorithm is more classical, which builds on the fact that the transport is one-dimensional, as noticed in Remark 1. We can thus use the algorithm of our own choice. In the following, we focus our attention on the limited downwind one. This can also be understood as a second longitudinal reconstruction.

\section{Numerical Results}

We present a few results obtained with the algorithm described above. Translation and rotation velocity fields are used. For all the test-cases, the spatial domain is $[0,1]^{2}$. The triangular mesh was generated by the software FreeFem++, see Fig. 7.

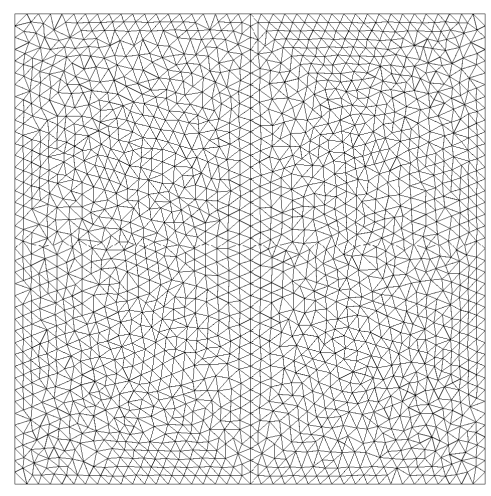

Fig. 7. Examplary Freefem++ mesh used for the numerical tests (here, 3766 triangles).

For all the test-cases, the Courant number

$$
\max _{j \in \mathbb{Z}} \frac{\Delta t}{s_{j}} \sum_{k \in K^{+}(j)} l_{j, k}\left(\mathbf{a}, n_{j, k}\right)
$$

takes the value 0.1 .

\subsection{Translation Field}

Translation of a square. Here we consider the velocity

$$
\mathbf{a}(t, x, y)=\left(\begin{array}{l}
1 \\
1
\end{array}\right) .
$$

This first test is the translation of the characteristic function of a square,

$$
u^{0}(x, y)=\chi_{[0.3,0.7]^{2}}(x, y) .
$$

The boundary conditions are periodic in $x$ and $y$. The initial condition and results at time $t=1$ (after one revolution) are reported in Fig. 8, and Fig. 9 reports the result for the upwind scheme. 

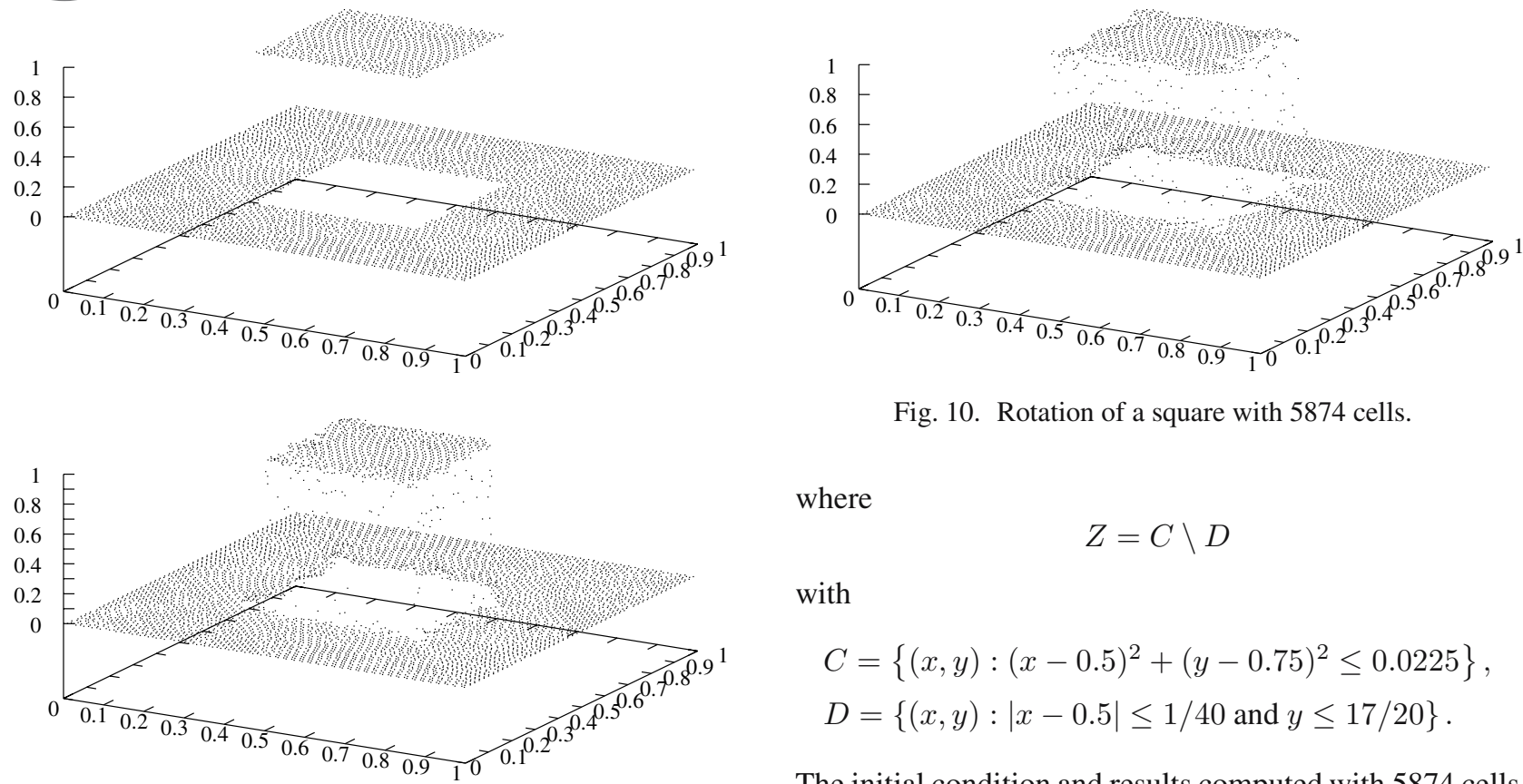

Fig. 10. Rotation of a square with 5874 cells.

where

$$
Z=C \backslash D
$$

with

$$
\begin{aligned}
& C=\left\{(x, y):(x-0.5)^{2}+(y-0.75)^{2} \leq 0.0225\right\}, \\
& D=\{(x, y):|x-0.5| \leq 1 / 40 \text { and } y \leq 17 / 20\} .
\end{aligned}
$$

Fig. 8. Translation of a square with 5874 cells.

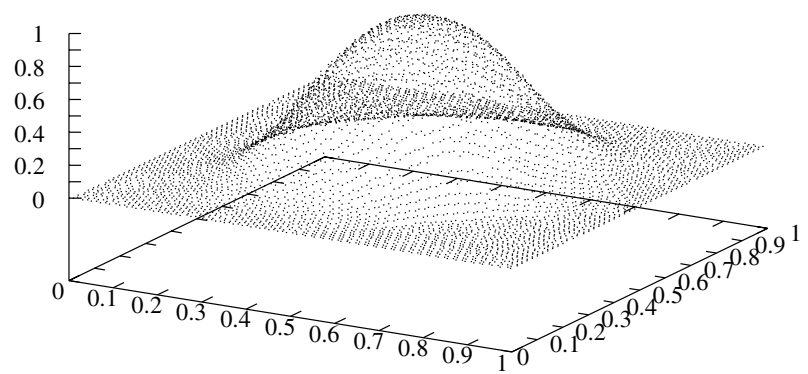

Fig. 9. Translation of a square with 5874 cells, the upwind scheme.

The initial condition and results computed with 5874 cells at time $t=1$ are displayed in Fig. 11. Then, Fig. 12 represents the same for a mesh made of 23618 cells, and Fig. 13 for a mesh composed of 94472 cells.

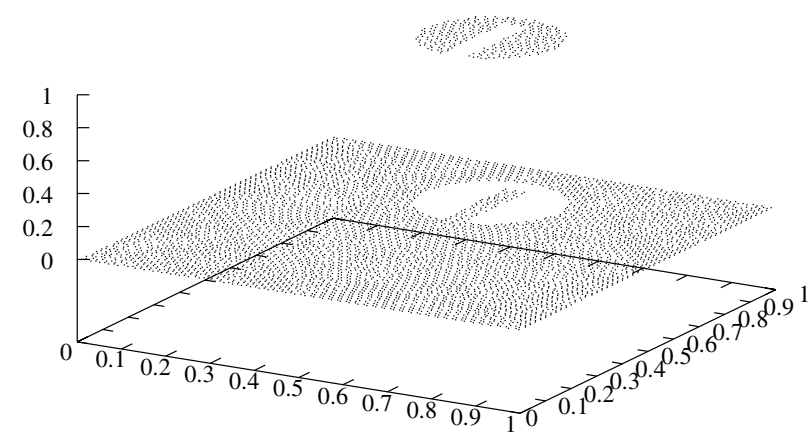

\subsection{Rotation Field}

Rotation of a square. Here the velocity field is

$$
\mathbf{a}(t, x, y)=\left(\begin{array}{c}
2 \pi y \\
-2 \pi x
\end{array}\right)
$$

and the initial condition is the same as in the preceding test. The final time is $t=1$. Figure 10 presents the result.

Zalesak's test case. The velocity field is the same as in the preceding test, i.e.,

$$
\mathbf{a}(t, x, y)=\left(\begin{array}{c}
2 \pi y \\
-2 \pi x
\end{array}\right)
$$

and the initial condition is taken from the original paper (Zalesak, 1979):

$$
u^{0}(x, y)=\chi_{Z}(x, y)
$$

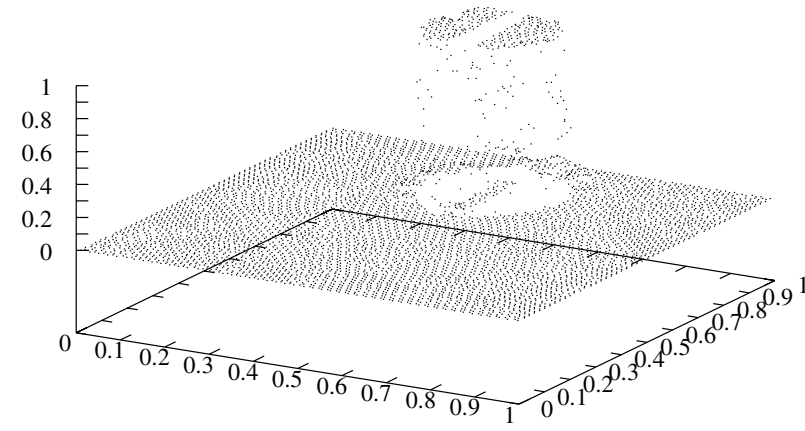

Fig. 11. Zalesak's test case with 5874 cells.

Deformation of a rectangle. The velocity field is now time varying:

$$
\mathbf{a}=\operatorname{sgn}(1-t)\left(\begin{array}{l}
3 \pi(y-0.5)^{2} \\
-3 \pi(x-0.5)^{2}
\end{array}\right),
$$




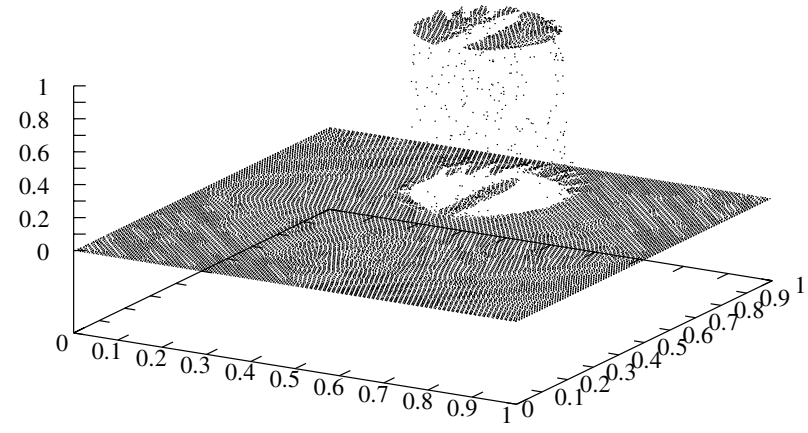

Fig. 12. Zalesak's test case with 23618 cells.

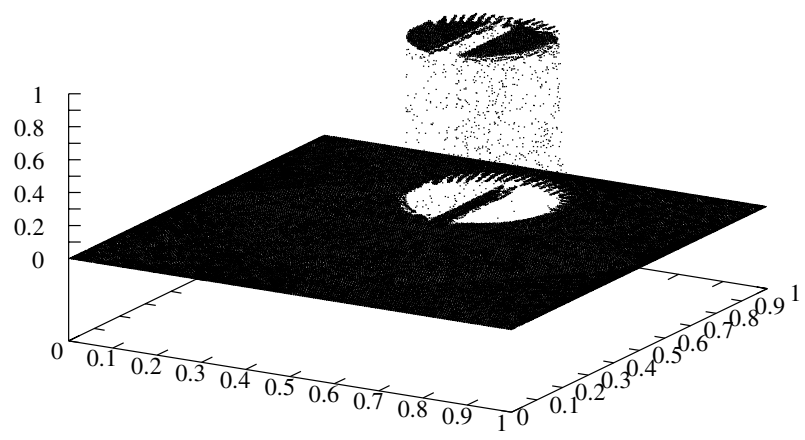

Fig. 13. Zalesak's test case with 94472 cells.

which means that at time $t=1$ the field is reversed. The initial condition is the characteristic function of a rectangle:

$$
u^{0}(x, y)=\chi_{[0.2,0.7] \times[0.45,0.55]}(x, y) .
$$

The exact solution at time 2 coincides with the initial condition.

We observe the initial condition (Fig. 14) and the results at time $t=1$ (Fig. 15) with 5874 cells and, finally, the result at time $t=2$ with 5874 cells (Fig. 16), 23618 cells (Fig. 17) and 94472 cells (Fig. 18). The result produced by the upwind scheme with 94472 cells is reported in Fig. 19.

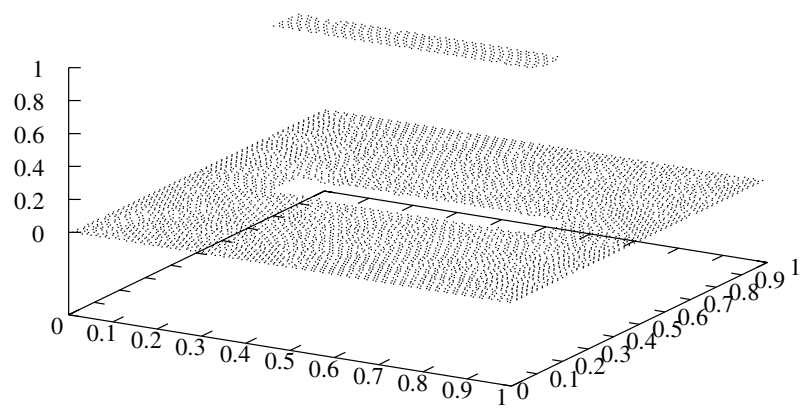

Fig. 14. Initial condition with 5874 cells.

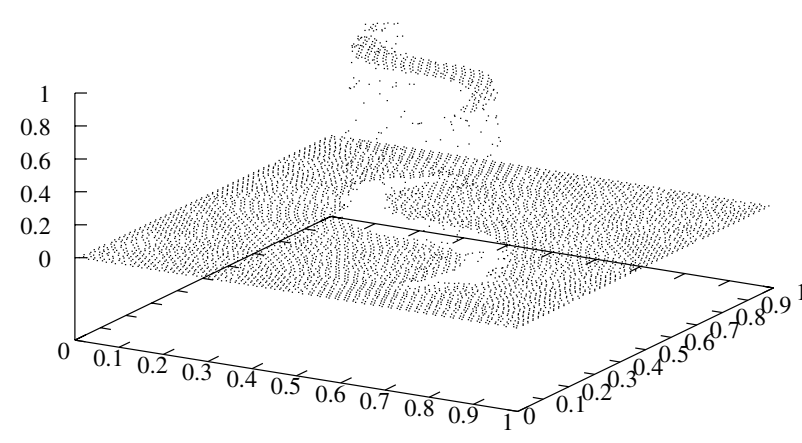

Fig. 15. Numerical solution with 5874 cells at time $t=1$.

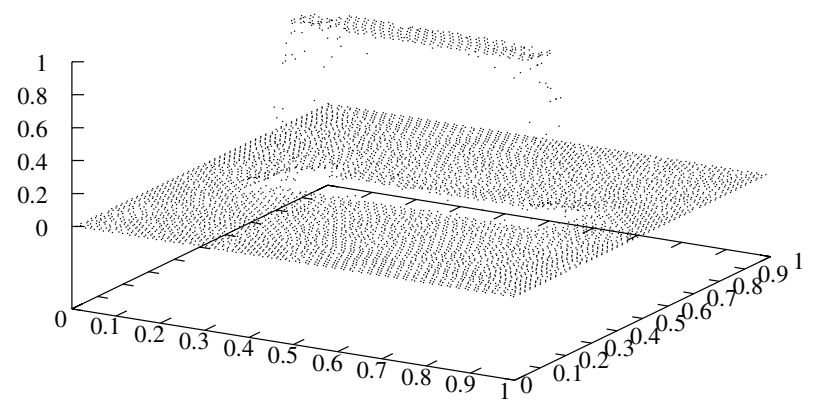

Fig. 16. Numerical solution with 5874 cells at time $t=2$.

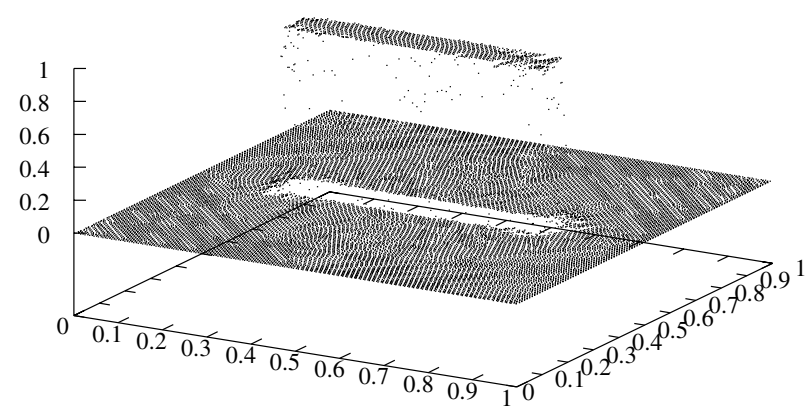

Fig. 17. Numerical solution with 23618 cells at time $t=2$.

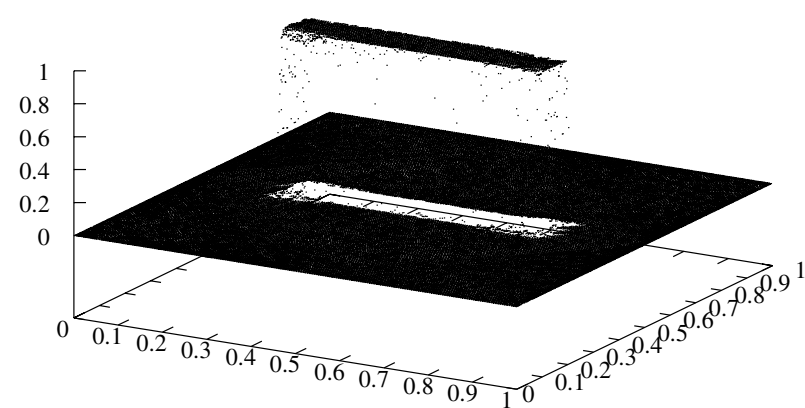

Fig. 18. Numerical solution with 94492 cells at time $t=2$. 


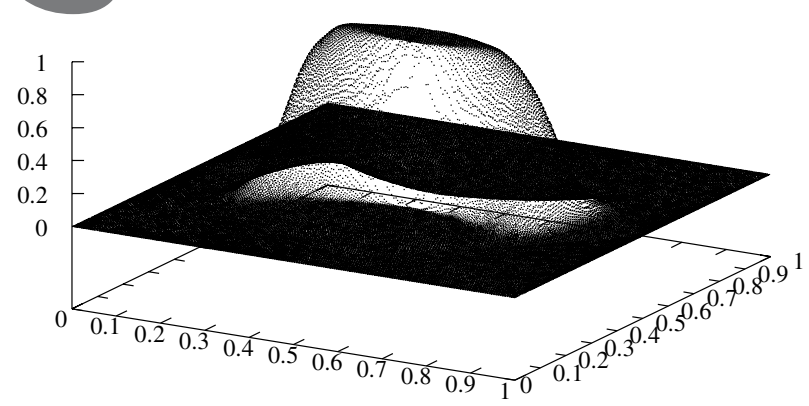

Fig. 19. Numerical solution with 94492 cells at time $t=2$, the upwind scheme.

\section{Final Comments}

We developed a new method for numerical transport in two dimensions. The approach is truly multidimensional in the sense that is does not refer to a one-dimensional reconstruction of interfaces. The numerical results show the anti-dissipative behaviour of the algorithm. The results are nevertheless not perfect. Indeed, the discontinuity lines may be degraded in a long time horizon (see Figs. 11-13).

The next study will concern the extension to general meshes (non-triangular) and three dimensions, as well as to an application to gas dynamics equations.

\section{References}

Després B. and Lagoutière F. (2001): Generalized Harten formalism and longitudinal variation diminishing schemes for linear advection on arbitrary grids. ESAIM: Mathematical Modelling and Numerical Analysis, Vol. 35, No. 6, pp. 1159-1183.
Després B. and Lagoutière F. (2001): Contact discontinuity capturing schemes for linear advection and compressible gas dynamics. Journal of Scientific Computing. Vol. 16, No. 4, pp. 479-524.

Després B. and Lagoutière F. (2007): Numerical resolution of a two-component compressible fluid model with interfaces. Progress in Computational Fluid Dynamics, (to appear).

Lagoutière F. (2005): Stability of reconstruction schemes for scalar hyperbolic conservation laws. Preprint available at http://www.ann.jussieu.fr/publications /2005/R05004.html.

Lagoutière F. (2006): Non-dissipative reconstruction schemes satisfying entropy inequalities. Preprint available at http: //www. ann.jussieu.fr/publications /2006/R06017.html.

Mosso S. and Cleancy S. (1995): A geometrical derived priority system for Young's interface reconstruction. Technical Report No. LA-CP-95-0081, Los Alamos National Laboratory.

Noh W.F. and Woodward P.R. (1976): SLIC (Simple Line Interface Calculation). Lecture Notes in Physics, Vol. 25, Berlin: Springer, pp. 330-339.

Youngs D.L. (1984): An interface tracking method for a $3 D$ eulerian hydrodynamics code. Technical Report No. 44/92/35, A.W.R.E. Aldermaston.

Zalesak S.T. (1979): Fully multidimensional flux-corrected transport algorithms for fluids. Journal of Computational Physics, Vol. 31, No. 3, pp. 335-362. 